\title{
Efficient Offline Outer/Inner DAC Mismatch Calibration in Wideband $\Delta \Sigma$ ADCs
}

Hui Liu, Pieter Rombouts, and Georges Gielen

This document is an author's draft version submitted for publication IEEE Trans. Circuits Syst.-I, The actual version was published as:

Liu, Hui, Pieter Rombouts, and Georges GE Gielen. "Efficient Offline Outer/Inner DAC Mismatch Calibration in Wideband ADCs." IEEE Transactions on Circuits and Systems I: Regular Papers 67.12 (2020): 4259-4269. 


\title{
Efficient Offline Outer/Inner DAC Mismatch Calibration in Wideband $\Delta \Sigma$ ADCs
}

\author{
Hui Liu, Pieter Rombouts, Member, IEEE, and Georges Gielen, Fellow, IEEE
}

\begin{abstract}
Distortion due to feedback DAC mismatch is a key limitation in $\Delta \Sigma$ ADCs for wideband wireless communications. This paper presents an efficient frequency-domain mask-based offline mismatch calibration method of both the outer DAC and the inner DACs in a $\Delta \Sigma$ ADC. The test stimulus for the calibration is a two-tone signal near the band edge. To avoid the need for high-performance signal generation, a frequency mask is applied to void the stimulus signal and its phase noise. In this way, the method is robust against distortion and jitter in the stimulus signal, which therefore could be combined from two lowquality signal generators. The two-tone band-edge signal has the additional benefit that the number of needed samples of the excitation signal is very modest because as many intermodulations as possible contribute to the calculation of the mismatch errors of the DACs. Experimental results confirming the calibration method are obtained from a prototype chip, designed for an $85 \mathrm{MHz}$ signal bandwidth in $28 \mathrm{~nm}$ CMOS technology. A two-tone stimulus around $78 \mathrm{MHz}$ is applied to calculate the mismatch of the outer DAC and the inner DAC with only $68 \mathrm{~K}$ samples. With the DACs calibrated, an SFDR improvement of $28.1 \mathrm{~dB}$ is achieved for a single-tone input at $5 \mathrm{MHz}$, while for a twotone input around $71 \mathrm{MHz}$, the IM3 is improved from -63.6 dBc to below the noise floor $(<-94.1 \mathrm{dBc})$. This illustrates the effectiveness of the approach.
\end{abstract}

Index Terms-Offline calibration, continuous-time, discretetime, wideband, $\Delta \Sigma$, analog-to-digital converter, DAC mismatch.

\section{INTRODUCTION}

$\mathbf{T}$ HE growth of mobile data is larger than that of the number of registered connections, which means that ever wider bandwidths (BW) are required for each wireless connection to integrate different radio bands [1], [2]. In the unavoidable presence of unwanted signals, including various blockers and other users, the analog-to-digital converter (ADC), as a critical block in the receiver system, needs to achieve not only the higher BW, but also a high linearity to suppress the harmonic and intermodulation (IM) distortions of these unwanted signals to below the minimum in-band signal [2], [3]. Multi-bit continuous-time (CT) $\Delta \Sigma$ ADCs with flat signal transfer function (STF) are widely applied in wireless communication systems for their good blocker immunity, inherent anti-aliasing and wide bandwidth [3]-[7].

Compared to single-bit CT $\Delta \Sigma$ ADCs like in [2], the limitation for multi-bit CT $\Delta \Sigma$ ADCs to achieve higher linearity is the component mismatch in the multi-bit digital-to-analog converters (DAC) in the feedback path [8]. As illustrative

This work has been supported by the National Science Foundation of Flanders.

H. Liu and G. Gielen are with the Department of Electrical Engineering, KU Leuven, B-3001 Leuven, Belgium (e-mail: hui.liu, gielen@esat.kuleuven.be).

P. Rombouts is with the Department of Electronics and Information Systems, Ghent University, 9000 Gent, Belgium (e-mail: pieter.rombouts@ugent.be).

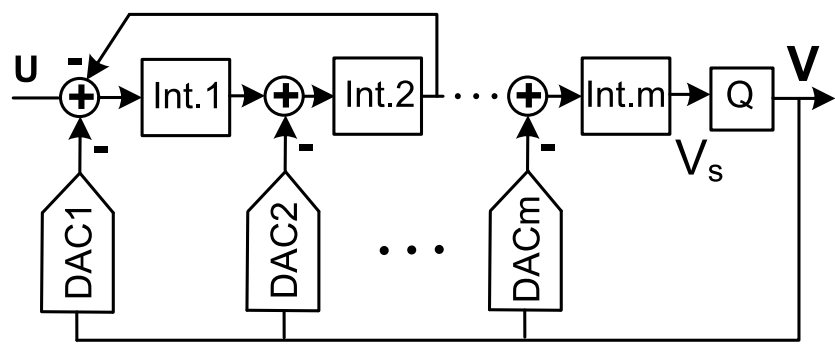

(a)

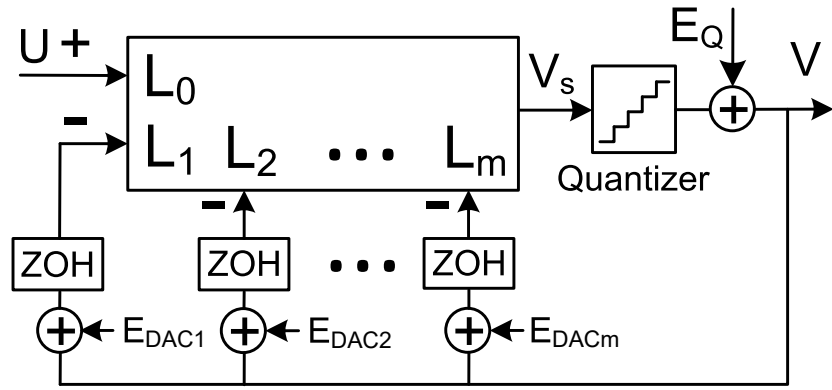

(b)

Fig. 1. (a) Block diagram of a general $m$-th-order CRFB $\Delta \Sigma$ ADC, and (b) its equivalent model, with the DACs' mismatch errors and the quantization error indicated.

architecture, Fig. 1(a) shows the block diagram of the cascadeof-resonators-with-feedback (CRFB) $\Delta \Sigma$ ADC topology. It has a low-pass STF (without peaking), which is known to achieve good blocker immunity and high in-band dynamic range in wireless communications [9]. When non-return-tozero (NTZ) DACs are used, the ADC's equivalent model is shown in Fig. 1(b), where the DACs' mismatch $\left(E_{D A C 1}\right.$ to $E_{D A C m}$ for an $m$-th-order $\Delta \Sigma \mathrm{ADC}$ ) and the zero-order hold $(\mathrm{ZOH})$ and quantization error $\left(E_{Q}\right)$ are added to the model. The mismatch of the outer $\mathrm{DAC}_{1}$ directly contributes to the digital output, causing distortion and limiting the dynamic range. While the mismatches of the inner DACs are suppressed by their prior stages, they do contribute to the digital output in case of wideband converters with low oversampling ratio (OSR), as will be shown below.

There are several ways to reduce the impact of the mismatch of the DACs in $\Delta \Sigma$ ADCs. The most intuitive way is to size up the cell unit of the DAC, which definitely increases the excess loop delay (ELD) of the closed-loop system, consumes more power and causes a larger dynamic error [4], [10]. Dynamic element matching techniques such as [11] have also been used to achieve high linearity. However, these techniques are less effective at low OSR and also increase the ELD, and therefore are not suitable for high-bandwidth designs. Several other methods that don't cause extra ELD, have been implemented in the analog domain. In [12], the current-copier technique 
has been proposed and verified on silicon [13], [14], but it suffers from charge leakage, charge injection and refresh spurs at low frequency [15]. To avoid the frequent refresh, a 1-bit DAC and DC value are used to only toggle two adjacent DAC elements and the DAC step size is calculated according to the digital output [16], [17]. The calculated mismatches of the DAC are calibrated through an injected analog compensation, which is a complicated the calibration procedure. Another similar technique is to measure the value of each DAC element, which requires extra complex mixed-signal circuitry [18]. The mismatch between the calculated digital value and the injected analog unit may also be problematic in [18]. In [19], a dithering-based technique has been used, but its performance was limited to a spurious-free dynamic range (SFDR) improvement of several dBs only.

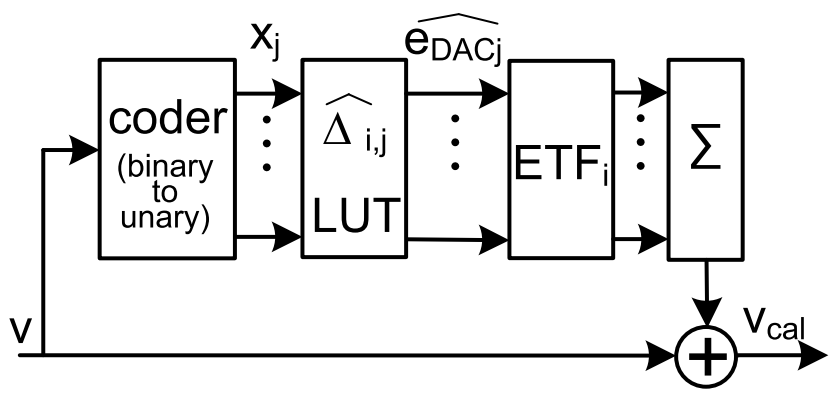

Fig. 2. Principle concept of digital calibration.

The mismatch of the DACs in a $\Delta \Sigma$ ADC can also be calibrated in the digital domain. As shown in Fig. 2, the calibration is performed by collecting estimated digital correction terms $\widehat{\Delta_{i, j}}$ from a look-up-table (LUT) and (after optional filtering with the corresponding error transfer function $\mathrm{ETF}_{\mathrm{i}}$ ) adding them to the modulator's digital output $V$ to obtain a calibrated output signal $V_{c a l}$. There are two families of digital calibration techniques. In the first family, the correction terms are obtained in an offline calibration cycle [20]-[22], while in the second family the correction terms are continuously updated online by some continuous adaptation technique [23] [25]. This work focuses on offline calibration techniques, which have the advantage that no modification at all of the analog modulator core is needed and that they can also be used for debug and evaluation purposes. When looking at related prior art, the time-domain techniques [20], [21] suffer from the fact that a high-quality low-jitter low-distortion signal generator is required for the calibration. In [22], an alternative frequency-domain method has been demonstrated, without the need of a high-quality signal generator. However, since a large portion of the DAC mismatch energy falls outside the band and is not used for the mismatch calculation, a very large number of samples is required for this method to significantly improve the performance, which is a challenge for wideband ADCs with high sampling speed. Our proposed method solves this issue.

For the inner DACs, such as $\mathrm{DAC}_{2}$ or $\mathrm{DAC}_{\mathrm{m}}$ in Fig. 1(a), it is widely assumed that their errors can be neglected, because their effect is reduced by the gain of the preceding integrators, and only recently authors have attempted to correct the corresponding errors. However, in wideband modulators, the OSR inevitably is very low, and hence the gain of prior integrators can be disappointingly low near the edge of the signal bandwidth. The inner DACs, especially the first inner DAC, can easily dominate the performance for higher input frequencies near the band edge, and therefore the errors of this inner DAC also need to be calibrated.

This paper therefore presents an efficient offline frequencydomain mask-based method to calibrate the mismatch of both the outer DAC and the inner DACs in wideband $\Delta \Sigma$ ADCs. The proposed method does not require an expensive signal generator nor too many data points. The effectiveness of the technique will be demonstrated on an experimental chip. Section II will illustrate the mechanism of DAC errors in $\Delta \Sigma$ ADCs. Section III will elaborate more about prior offline calibration techniques. The proposed offline calibration method will be explained in Section IV. Simulation and experimental results will be given in Section V and in Section VI, respectively. The paper will be concluded in Section VII.

\section{DAC ERROR BEHAVIOR}

\section{A. Error Transfer Function}

The error transfer function (ETF) is the transfer function from the mismatch error of one of the feedback DACs to the digital output. To explain the method, we start with the cascade of resonators with feedback (CRFB) structure of Fig. 1. The output signal $V$ for the system of Fig. 1(b) can be written as ${ }^{1}$ :

$$
\begin{gathered}
V=S T F \cdot U+N T F \cdot E_{Q}-\sum_{i=1}^{m}\left(E T F_{i} \cdot E_{D A C i}\right) \\
\left\{\begin{array}{l}
S T F=\frac{L_{0}}{1+\sum_{i=1}^{m} L_{i}} \\
N T F=\frac{1}{1+\sum_{i=1}^{m} L_{i}} \\
E T F_{i}=N T F \cdot L_{i}
\end{array}\right.
\end{gathered}
$$

where $L_{0}$ and $L_{i(1-m)}$ are the $z$-domain expression of the transfer function from the ADC input $U$ to the node $V_{s}$ in front of the quantizer, and the $z$-domain expression of the transfer function from $D A C_{i(1-m)}$ to the node $V_{s}$ in Fig. 1(a), respectively. NTF is the noise transfer function of the $\triangle \Sigma$ ADC for the quantization noise. From (1) it is clear that, if $E T F_{i}$ and $E_{D A C i}$ are known for all DACs, the effect of DAC mismatch can be eliminated from the digital output of the $\Delta \Sigma$ ADC by subtracting suitable correction terms. In the past, many authors have used the simplifying assumption that only the first DAC's errors should be corrected and additionally that the first error transfer function $E T F_{1}$ is equal to a constant (typically unity) across the entire band. While both assumptions are approximately valid for the case of a high OSR, they are incorrect in the wideband case (where the OSR is low), as we will demonstrate below.

To improve the quantization noise shaping performance, typically local feedback resonators are used [8]. However when doing this, the ETF is no longer constant, neither for the cascade of resonators with feedforward (CRFF) topology nor for the CRFB topology. To investigate the ETF for an effective example, we illustratively study a fourth-order CRFB 


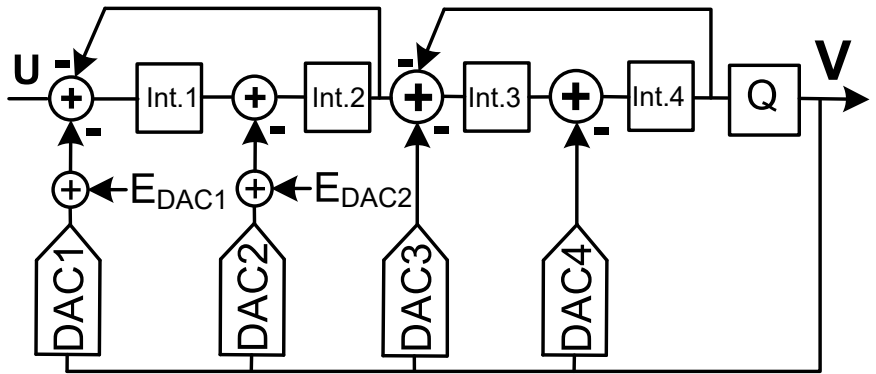

Fig. 3. Case study of a 4th-order CRFB $\Delta \Sigma$ ADC, with mismatch in both the outer $\mathrm{DAC}_{1}$ and the inner $\mathrm{DAC}_{2}$ indicated.

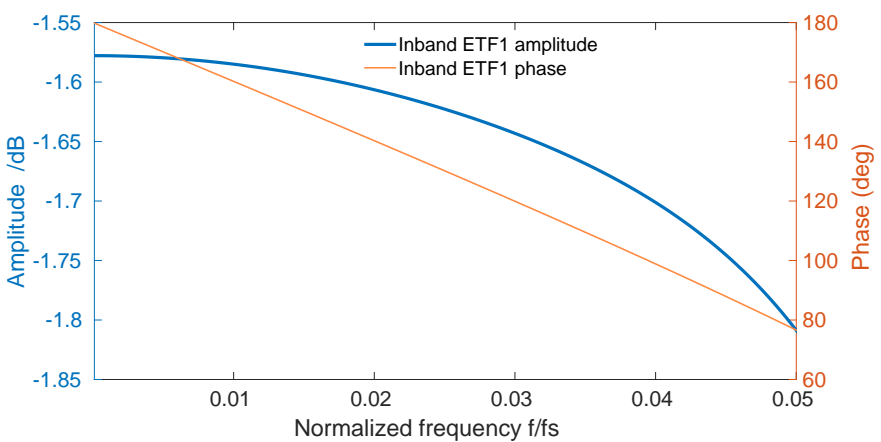

Fig. 4. The in-band amplitude and phase of the ETF of the DAC $\mathrm{D}_{1}$ mismatch for the $\Delta \Sigma$ ADC of Fig. 3.

$\Delta \Sigma$ ADC with 4-bit quantizer, shown in Fig. 3, where also the $\mathrm{DAC}_{1}$ mismatch is indicated. The in-band ETF for the nominal modulator design (with ideal integrators) is illustrated in Fig. 4 for an $\mathrm{OSR}=10$. The amplitude of $\mathrm{ETF}_{1}$ shows a modest fluctuation of about $0.2 \mathrm{~dB}$, but the phase of $\mathrm{ETF}_{1}$ varies significantly: from 180 degrees to 76.7 degrees. With finite opamp gain and bandwidth included, the fluctuation can be larger.

The mismatch of $\mathrm{DAC}_{1}$, without any calibration, results in significant distortion, as shown in Fig. 5. When $\mathrm{ETF}_{1}$ is approximated as a constant $(0.83=-1.62 \mathrm{~dB})$ like in [20] and [22], the calibrated spectrum, with the correct DAC mismatch values, is shown in Fig. 5(a). With the approximated constant ETF $_{1}$ of 0.83 the calibrated output is given by:

$$
V_{c a l}=S T F \cdot U+N T F \cdot E_{Q}+\left(0.83-E T F_{1}\right) \cdot E_{D A C 1}
$$

Since $\mathrm{ETF}_{1}$ in reality is not exactly a constant, as shown in Fig. 4 , the output spectrum $V_{c a l}$ can not perfectly be reconstructed and has large distortions, which was also observed in [20] and [22]. Doing the calibration with the actual $\mathrm{ETF}_{1}$ and the exact mismatch values of $\mathrm{DAC}_{1}$, the ideal output spectrum is obtained, as shown in Fig. 5(b). Hence, in addition to using an accurate estimate of $E_{D A C}$, it is also important to use the correct ETF both in amplitude and phase, especially for the CRFB topology (Fig. 1), where the phase shifts drastically. Note that these ETFs in principle are known (i.e. they are defined by the loop filter design). However, due to circuit imperfections such as finite opamp bandwidth and parasitic loop delay, the actual ETF can deviate from its nominal case. We will come back to this in section VI.

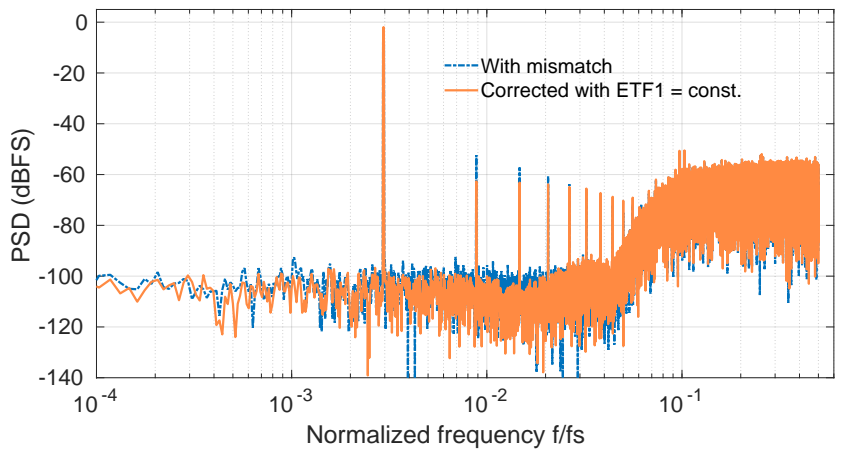

(a)

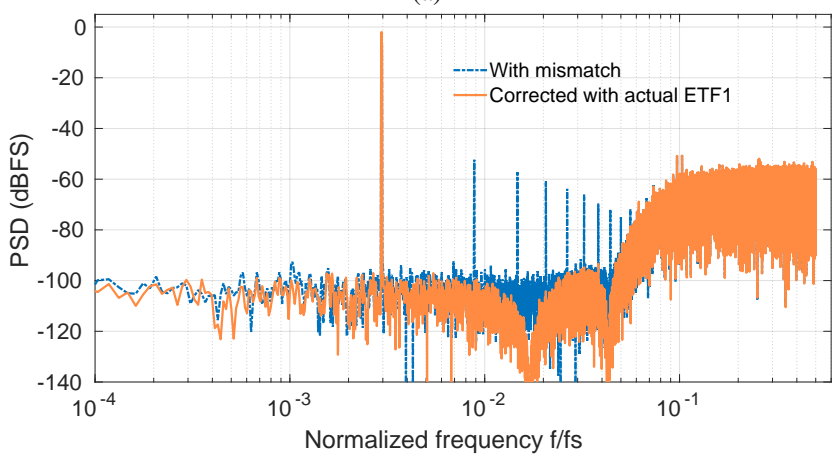

(b)

Fig. 5. Output spectra of the CRFB topology of Fig. 3 for the case where there is only $\mathrm{DAC}_{1}$ mismatch without calibration compared to: (a) correction with the approximation of $\mathrm{ETF}_{1}=0.83$, and (b) correction with the actual $\mathrm{ETF}_{1}$.

\section{B. DAC error}

We assume that all the DACs in Fig. 1 have $k$ elements (for a $n$-bit quantizer: $k=2^{n}-1$ ), which are driven by control signals $X_{j}$ derived from the output of the quantizer $V$ in such a way that:

$$
V=\sum_{j=1}^{k} X_{j}
$$

Each control signal can take two values, depending on whether the element is selected or not. For a differential circuit, we adopt the most convenient notation by using +1 or -1 for $X_{j}$. Then the $\mathrm{DAC}_{\mathrm{i}}$ error $e_{D A C i}$ can be expressed as:

$$
E_{D A C i}=\sum_{j=1}^{k} \Delta_{i, j} X_{j}
$$

where $\Delta_{i, j}$ is the mismatch of the $j t h$ element of $\mathrm{DAC}_{\mathrm{i}}$. The offset of the DAC element is omitted for simplicity, because it only generates an overall offset, which is a less critical concern [4]. The control signals $X_{j}$ are known from the quantizer output $V$, hence to estimate the DAC error $E_{D A C}$, only the element mismatches $\Delta_{i, j}$ need to be estimated.

\section{Offline calibration core idea}

The general flow of an offline calibration consists of the following steps. First, the ADC is stimulated by some input stimulus signal and the corresponding output signal $V$ is collected. Then in the second step the input signal as well as (most) of the quantization noise is removed, either in the 
time domain or in the frequency domain, from $V$ to obtain a measured error signal $V_{\text {err }}$. From (1) and (5), we immediately see that:

$$
V_{\text {err }}=-\sum_{i=1}^{m} E T F_{i} \sum_{j=1}^{k} \Delta_{i, j} X_{j}+N_{c a l}
$$

Here $N_{\text {cal }}$ refers to noise that is uncorrelated to the DAC errors. This noise may come from circuit noise during the measurements, but also from incompletely removed quantization noise. In (6), the signals $V_{e r r}$ and $X_{j}$ are known after the calibration cycle. If there would be no noise, the mismatches $\Delta_{i, j}$ can then be computed by solving directly the above equation. In the presence of noise, the mismatches $\Delta_{i, j}$ can typically be found by solving this equation by minimizing its mean square error.

\section{Prior Offline Calibration Methods}

With regard to the core calibration approach described above, prior art methods differ in two aspects: 1) in the choice of the input stimulus, and 2) in the way how the input signal and the quantization noise are removed from the measurement.

The first implementations of this approach were the sinusoidal-signal-based time-domain calibration techniques of [20], [21], which have been applied to several state-of-theart designs [26], [27]. For these methods in-band sinusoidal signals, that are large enough to activate all the DAC elements, are fed to the ADC. Then the input stimulus signal is removed by curve fitting in the time domain, and the estimation of the $\Delta_{i, j}$ is obtained by minimizing the time-domain error.

Unfortunately, these time domain methods are computationally intensive due to the need to perform time-domain curve fitting and filtering [20], [21]. Moreover, they are sensitive to harmonic distortion and, even worse, jitter or phase noise present in the input signal. This is shown in Fig. 6(a) where the simulated results are shown for the case of an input signal with jitter. For the simulations, a 4-bit, 4th-order CRFF synthesized with an $H_{\infty}=2$ and an OSR=10 was used [8], [28]. Note that the figure uses a Hann window in such a way that the window effect is entirely cancelled and the (still relatively narrow) line width seen in the figure is due to the intentionally added jitter. It is clear from Fig. 6(a) that the calibration of [20], [21] fails catastrophically, which is due to the fact that the algorithm tries to find correction coefficients that cancel the phase noise in the used input signal.

In [22], an alternative technique is proposed based on the frequency-domain processing of an out-of-band signal, to avoid the need for a high-linearity low-jitter input signal. Unfortunately, this technique requires a large number of samples to do the calibration. To illustrate this, we performed this calibration technique where we used a sinusoidal input signal just outside the signal band. First, we carried out the calibration with the same number of data points (i.e. $40 \mathrm{~K}$ ) as in the other cases. The result for this case is shown in Fig. 6(b). It is clear that the result is poor and that, although the noise floor is decreased, the distortion actually gets worse. When a larger number of samples is used, the accuracy of [22] gets better. For example, when $10 \mathrm{M}$ data points are used in the calibration, the

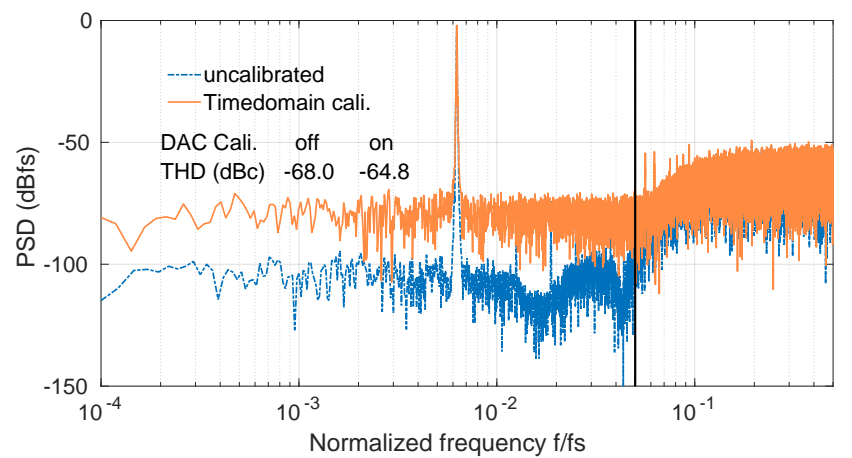

(a)

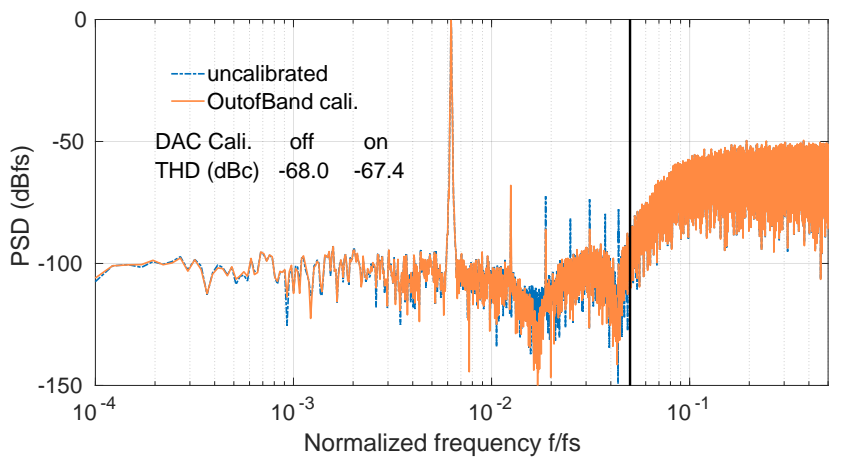

(b)

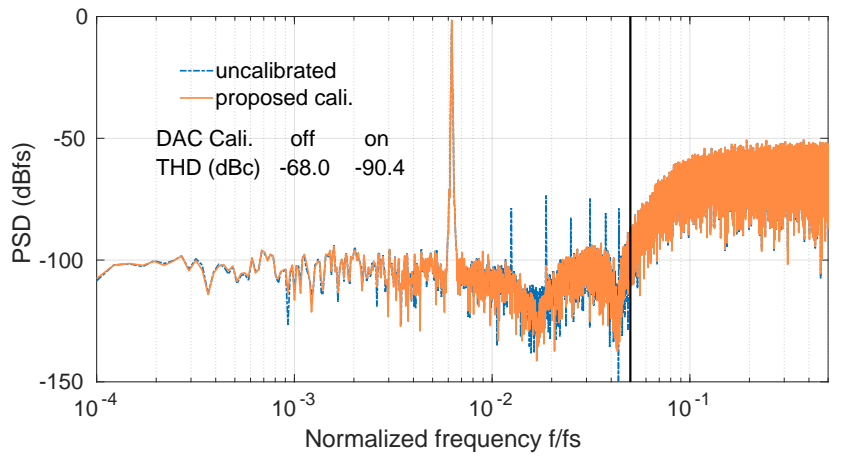

(c)

Fig. 6. Comparison of the calibration techniques for the case of an input signal with jitter (40K FFTs). (a) Time-domain technique of [20]; (b) out-ofband excitation technique of [22]; (c) proposed mask-based frequency-domain technique.

distortions of nine DAC elements reduce to about $-100 \mathrm{dBc}$, which is still significantly less good than for our proposed new method with only $40 \mathrm{~K}$ data points (Fig. 6(c)). An additional pitfall of [22] is that the method is not elaborated for the case where ETF is not a constant. Furthermore, despite the inherent anti-aliasing of a continuous-time $\Sigma \Delta$ modulator, when the OSR is very low, aliased harmonics of the out-ofband stimulus may also cause in-band spurs, partially offseting the main advantage of the technique that no accurate signal source is needed. These disadvantages are overcome in our method.

\section{Proposed Calibration Method}

\section{A. Input Stimulus selection}

For the case of wideband $\Delta \Sigma$ ADCs with a low OSR, the band-edge gain of all integrators in the feedback loop 


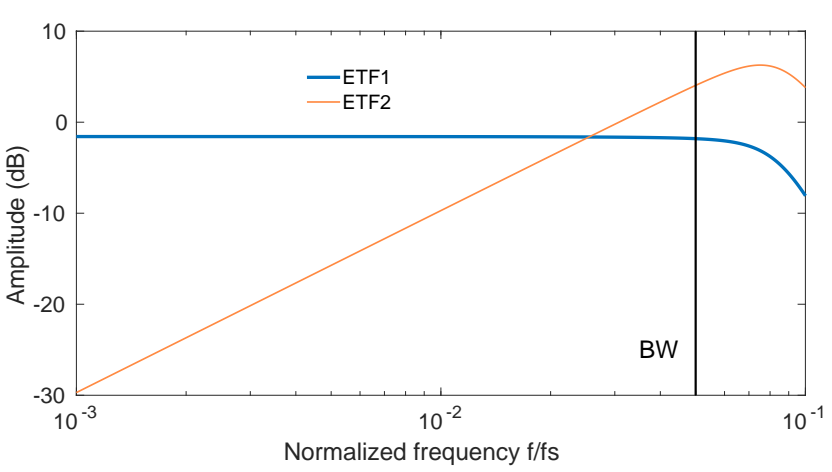

Fig. 7. Magnitude plot of the ETF of the mismatch for the outer $\mathrm{DAC}_{1}$ and the inner $\mathrm{DAC}_{2}$ in the architecture of Fig. 3, with $\mathrm{ETF}_{2}$ becoming large near the signal band edge.

inevitably becomes relatively low. This is particularly the case for the first integrator in the CRFB topology in Fig. 3 [4], [8]. For example, for the illustrative case of a 4-bit, 4th order CRFB topology with $H_{\infty}=2$ and OSR=10, the bandedge gain is below zero $\mathrm{dB}$, which leads to an $\mathrm{ETF}_{2}$ gain of $4 \mathrm{~dB}$ at the band edge, as shown in Fig. 7. Therefore, the common assumption that the mismatch of $\mathrm{DAC}_{2}$ can be neglected is definitely incorrect in this case. This mismatch manifests itself clearly near the bandwidth edge and also needs to be calibrated. While the first integrator gain is very low in the CRFB topology, the other integrator coefficients are significantly larger (e.g. in our numerical example the second integrator gain is more than $10 \mathrm{~dB}$ higher than that of the first one). As a consequence, the input-referred distortion of the third and later DACs indeed do not limit the ADC performance, but the mismatch of $\mathrm{DAC}_{2}$ becomes important. Hence, we will elaborate in this paper the mismatch calibration of the outer $\mathrm{DAC}_{1}$ and of the first inner DAC, i.e. $\mathrm{DAC}_{2}$. The method can straightforwardly be extended to the calibration of more than two DACs.

The model of the CRFB $\Delta \Sigma$ ADC of Fig. 3 was built with Schreier's well known toolbox [8], [28]. It has resonators to optimize the zeros location to achieve maximum signal-toquantization-noise ratio (SQNR). The ADC has a 4-bit quantizer, an OSR $=10$, and $H_{\infty}=2$, achieving a corresponding bandwidth of $85 \mathrm{MHz}$. As shown in Fig. 7, the amplitude of the mismatch ETF of DAC 1 remains almost constant, slightly smaller than 1, across the signal bandwidth, while that of $\mathrm{DAC}_{2}$ behaves as a first-order differentiation (increasing with $20 \mathrm{~dB} /$ decade) within the bandwidth. The mismatch of $\mathrm{DAC}_{2}$ is therefore attenuated at low frequencies. When the frequency increases, the $\mathrm{ETF}_{2}$ magnitude becomes larger than $0 \mathrm{~dB}$. Therefore, the mismatch of $\mathrm{DAC}_{2}$ dominates the distortion in the upper part of the bandwidth (in this example above $50 \mathrm{MHz}$ ).

While the proposed calibration technique (see below) can work with a sinewave excitation, as was done for Fig. 6(c), this is not the best choice. Ideally, we want an input signal that does not put any requirements on the signal generator in terms of low distortion or jitter. To also efficiently calibrate the inner DAC(s), a significant part of the DAC-induced distortion should be in the upper part of the signal band. To alleviate

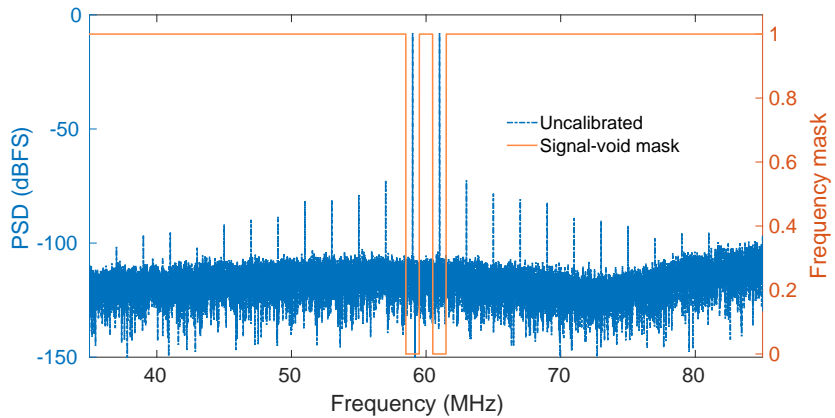

(a)

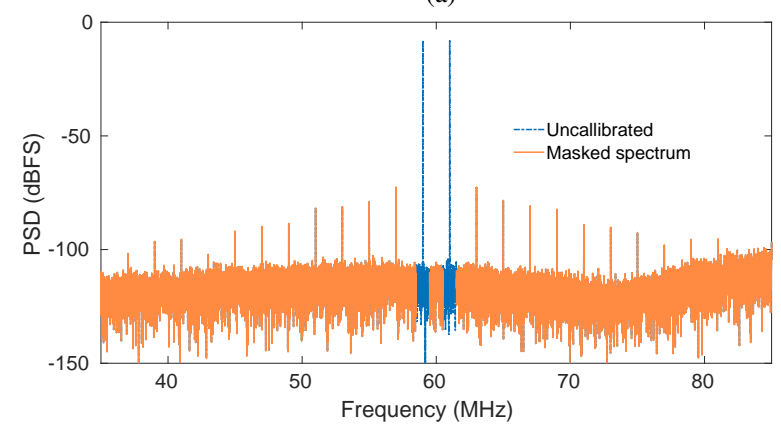

(b)

Fig. 8. (a) The uncalibrated output spectrum and the frequency-domain mask used, and (b) the masked uncalibrated spectrum.

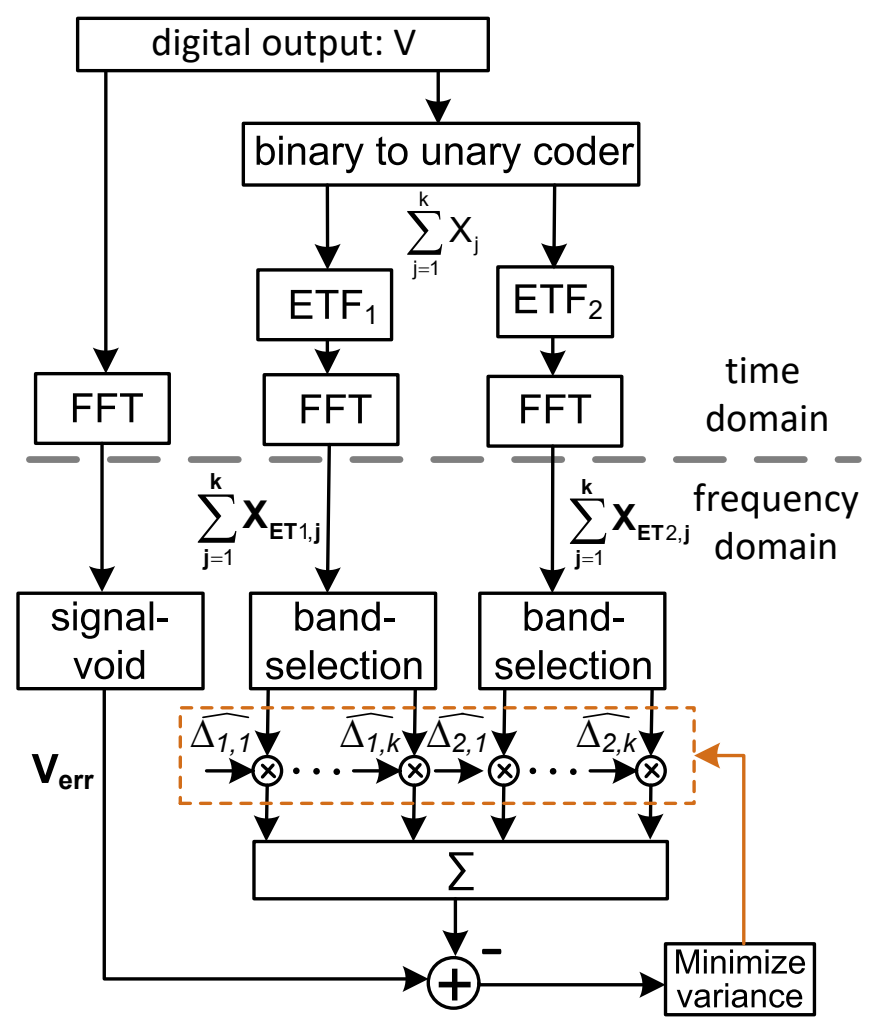

Fig. 9. Schematic representation of the proposed calibration technique.

this, we propose to use a closely spaced two-tone excitation, that could equally well be generated with two independent low-quality signal sources of which the outputs are combined. The frequency of the two-tone signals should be above half the bandwidth, such that all harmonic distortions of the signal 
generator are outside the bandwidth. With such an input signal, DAC mismatches will manifest themselves as intermodulations falling within the bandwidth. This is shown in Fig. 8(a), where our example $85 \mathrm{MHz}-\mathrm{BW}$ CRFB modulator is excited with two -8dBFS input tones at $59 \mathrm{MHz}$ and $61 \mathrm{MHz}$. With this choice, all the DAC elements are activated and most intermodulation components are within the bandwidth of $85 \mathrm{MHz}$.

\section{B. Frequency-Domain Mask-based Calibration}

The actual calibration calculations are carried out in the frequency domain. Basically this is done by constructing an FFT approximation of (6). A schematic representation of the overall calibration approach is shown in Fig. 9.

The first step is to take the FFT of the digital output signal $v(n)$ (after proper windowing e.g. with a Hann or Blackman window) [29]. This provides the FFT result $\mathbf{V}$, which we represent as a row vector over the discrete frequency values.

To remove the out-of-band quantization noise and the input signal, a frequency-domain signal-void mask is applied. If the input stimuli have in-band imperfections, such as phase noise, the mask can also be used to remove these. With our choice of a two-tone stimulus near the band edge, phase noise is normally the only in-band imperfection, which is readily removed by the signal-void mask $M_{s}(f)$ defined as follows:

$$
\begin{array}{rlrl}
M_{s}(f) & =0, & \forall f>B W \text { and } \quad \forall f \approx f_{\text {sig }} \\
& =1, \quad \text { elsewhere }
\end{array}
$$

The signal-void mask $M_{s}(f)$ is shown in Fig. 8(a). The window of the mask, i.e. where $M_{s}(f)$ is 0 near the input frequencies, should properly attenuate the non-ideal input signal, such as phase noise, while keeping all the intermodulation components. To maintain a consistent notation, we will use the notation $\mathbf{M}_{\mathbf{s}}$ for the row vector containing the mask evaluated on the same frequency grid as the FFT signal V. The masked output spectrum $\mathbf{V}_{\text {err }}$ is then:

$$
\mathrm{V}_{\text {err }}=\mathrm{V} \cdots \mathbf{M}_{\mathbf{s}}
$$

where..$\quad$ is used to denote the element-wise multiplication. This masked output spectrum $\mathbf{V}_{\text {err }}$ corresponds to the FFT of $V_{e r r}$, see Eq. (6), and is shown in Fig. 8(b).

The DAC element control signal, $X_{j}\left(x_{j}(n)\right.$ in the time domain) are filtered by both $\mathrm{ETF}_{1}$ and $\mathrm{ETF}_{2}$, which are assumed to be known or simulated, and then the corresponding FFTs are applied, leading to:

$$
\begin{aligned}
& \mathbf{X}_{\mathbf{E T} 1, \mathbf{j}}=\operatorname{FFT}\left\{x_{j}(n) * \operatorname{etf}_{1}(n)\right\} \\
& \mathbf{X}_{\mathbf{E T} 2, \mathbf{j}}=\operatorname{FFT}\left\{x_{j}(n) * \operatorname{etf}_{2}(n)\right\}
\end{aligned}
$$

Here, $*$ stands for the convolution, and etf $f_{1}$ and $\mathrm{etf}_{2}$ stand for the impulse response of the respective error transfer functions. Again, each Fourier transform is represented as a column vector over the frequency grid. To remove the out-of-band components, the band-selection mask $M_{b}$ is defined as follows:

$$
\begin{aligned}
M_{b}(f) & =0, & & \forall f>B W \\
& =1, & & \text { elsewhere }
\end{aligned}
$$

Again, the notation $\mathbf{M}_{\mathbf{b}}$ is used for the row vector containing the mask evaluated on the same frequency grid as the FFT signals. Now, we obtain the band-limited FFTs $\mathbf{X}_{\mathbf{E T b} \mathbf{b}, \mathbf{j}}$ and $\mathbf{X}_{\mathbf{E T b 2 , i}}$ of the filtered selection signals:

$$
\begin{aligned}
& \mathbf{X}_{\text {ETb1,j }}=\mathbf{X}_{\mathbf{E T} 1, \mathbf{j}} \cdots \mathbf{M}_{\mathbf{b}} \\
& \mathbf{X}_{\mathbf{E T b 2 , i}}=\mathbf{X}_{\mathbf{E T 2}, \mathbf{j}} \cdots \mathbf{M}_{\mathbf{b}}
\end{aligned}
$$

Then we define the total $\mathbf{X}_{\mathbf{t}}$ matrix, by combining the FFT column vectors for both $\mathrm{DAC}_{1}$ and $\mathrm{DAC}_{2}$ as follows:

$$
\mathbf{X}_{\mathbf{t}}=\left[\mathbf{X}_{\mathbf{E T b 1 , 1}} \cdots \mathbf{X}_{\mathbf{E T b 1 , k}} \mathbf{X}_{\mathbf{E T b 2 , 1}} \cdots \mathbf{X}_{\mathbf{E T b 2 , k}}\right]
$$

where $k$ stands for the number of DAC elements. The mismatch vector of the two DACs, $\mathbf{W}$, is combined as:

$$
\mathbf{W}=\left[\begin{array}{llllll}
\widehat{\Delta_{1,1}} & \cdots & \widehat{\Delta_{1, k}} & \widehat{\Delta_{2,1}} & \cdots & \widehat{\Delta_{2, k}}
\end{array}\right]^{T}
$$

where $\widehat{\Delta_{i, j}}$ represents the estimated mismatch value to be distinguished from the actual real mismatch value $\Delta_{i, j}$. Next, the FFT-domain version of (6) for the case that there is no other noise can be written as:

$$
\mathbf{X}_{\mathbf{t}} \cdot \mathbf{W}=\mathbf{V}_{\text {err }}
$$

Now, the mismatch vector $\Delta$ can be found by solving this system of complex equations (remember that the FFTs have both real and imaginary components). The number of equations in this system is equal to the number of baseband frequency points $(7.2 \mathrm{~K}$ equations in the examples below), while the number of unknown elements in $\mathbf{W}$ is the total number of DAC elements ( $2 k=30$ in the examples below). Furthermore, due to the presence of noise (due to the residue quantization noise or other noise), this system is always overdetermined and needs to be solved in a least mean square way, with the mathematical constraint that $\Delta$ should be real. Straightforward algebraic manipulation similar to [20], [22] shows that the solution is equivalent to solving the linear system of equations:

$$
\left[R e\left(\mathbf{X}_{\mathbf{t}}^{\mathbf{H}} \mathbf{X}_{\mathbf{t}}\right)\right] \cdot \mathbf{W}=\operatorname{Re}\left(\mathbf{X}_{\mathbf{t}}^{\mathbf{H}} \mathbf{V}_{\mathbf{e r r}}\right)
$$

where $\mathbf{X}_{\mathbf{t}}^{\mathbf{H}}$ stands for the conjugate transpose of $\mathbf{X}_{\mathbf{t}}$

Once the mismatches $\widehat{\Delta_{i, j}}$ are known, they can be stored in a LUT. During normal $\triangle \Sigma$ ADC operation, an accurate estimation $e_{\overline{D A C} i}$ of the DAC error is obtained as $\sum_{j=1}^{k} \widehat{\Delta_{i, j}} x_{j}(n)$, and the digital output of Fig. 3 can be corrected as:

$$
\begin{array}{r}
V+\sum_{i=1}^{2}\left(E T F_{i} \cdot \widehat{E_{D A C i}}\right)=S T F \cdot U+N T F \cdot E_{Q} \\
+\underbrace{\sum_{i=1}^{2}\left(E T F_{i} \cdot\left(\widehat{E_{D A C i}}-E_{D A C i}\right)\right)}_{\approx 0}
\end{array}
$$

Clearly, the error due to mismatch of the two feedback DACs is almost eliminated.

\section{Simulation Results}

To illustrate the effectiveness of the proposed method, it has been applied to the CRFB model of Fig. 3. $\mathrm{DAC}_{1}$ and $\mathrm{DAC}_{2}$ are given mismatches with a standard deviation $(\sigma)$ of $0.3 \%$ and a standard deviation of $0.5 \%$, respectively. The calibration has been performed with the excitation signal shown in Fig. 


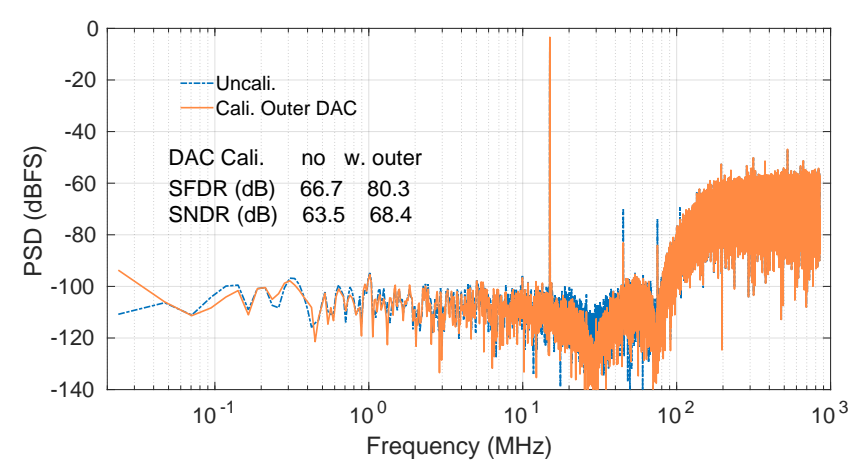

(a)

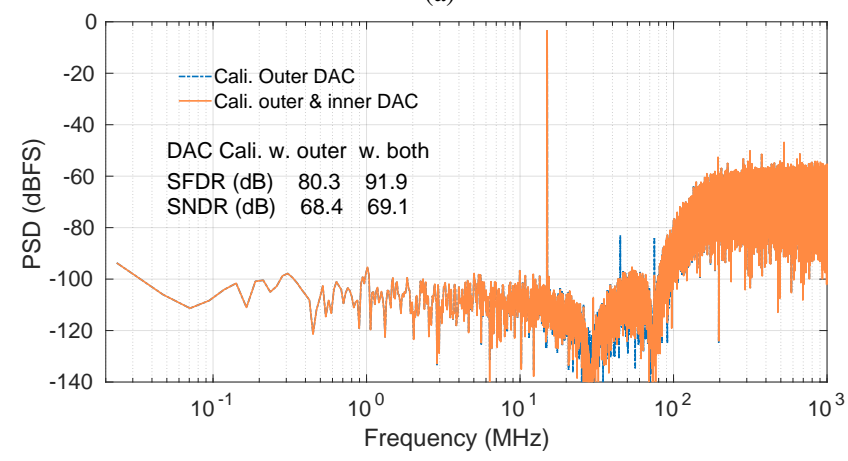

(b)

Fig. 10. Simulated single-tone spectra: (a) with only the outer $\mathrm{DAC}_{1}$ calibrated, and (b) with both the outer $\mathrm{DAC}_{1}$ and the inner $\mathrm{DAC}_{2}$ calibrated. The latter case has no residual distortion.

8. A simulated in-band sequence of $68 \mathrm{~K}$ samples is used for the calculation, and the exact ETFs, known through simulation, are used. For a $-3.4 \mathrm{dBFS} 15 \mathrm{MHz}$ input signal, the uncalibrated spectrum with 72K-point FFT is shown in Fig. 10(a). Due to the DACs' mismatch, the SFDR and SNDR are limited to $66.7 \mathrm{~dB}$ and $63.5 \mathrm{~dB}$, respectively. The estimated values are calculated using equation (12). When only the outer $\mathrm{DAC}_{1}$ is calibrated, the calibrated spectrum is presented in Fig. 10(a), where both the SFDR and SNDR are improved. However, the distortion due to the inner $\mathrm{DAC}_{2}$ is still visible in the spectrum. The result after calibrating both the outer $\mathrm{DAC}_{1}$ and the inner $\mathrm{DAC}_{2}$ is shown in Fig. 10(b), where the SFDR and SNDR are further improved to $91.9 \mathrm{~dB}$ and $69.1 \mathrm{~dB}$, respectively.

In addition, the calibration has been applied to the case of a band-edge two-tone input signal with two -8.7dBFS input tones at $\mathrm{f}_{1}=77 \mathrm{MHz}$ and $\mathrm{f}_{2}=79 \mathrm{MHz}$, respectively. For this, the same calculated mismatch values obtained from the calibration shown in Fig. 8 were used. The results are depicted in Fig. 11. With the calibration of the outer $\mathrm{DAC}_{1}$, IM3 is $76.0 \mathrm{dBc}$ at $75 \mathrm{MHz}\left(2 \mathrm{f}_{1}-\mathrm{f}_{2}\right)$ and is $-75.6 \mathrm{dBc}$ at $81 \mathrm{MHz}$ $\left(2 \mathrm{f}_{2}-\mathrm{f}_{1}\right)$. When both the outer $D A C_{1}$ and the inner $\mathrm{DAC}_{2}$ are calibrated, IM3 is further improved to $-103 \mathrm{dBc}$ at $75 \mathrm{MHz}$ and to $-91.8 \mathrm{dBc}$ at $81 \mathrm{MHz}$. Clearly, the distortion is reduced hugely.

\section{EXPERIMENTAL RESULTS}

To illustrate the method on real silicon, the above described calibration technique has been applied to a fabricated continuous-time $\Delta \Sigma$ modulator chip for wideband wireless communication that was available to the authors, with topology

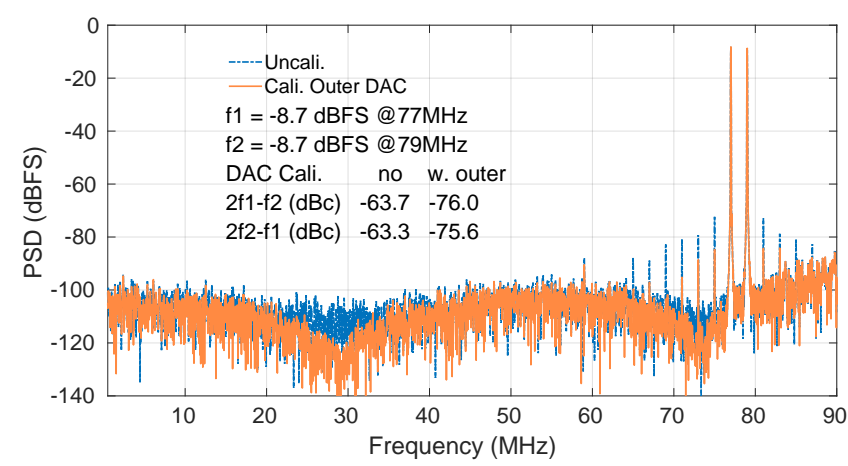

(a)

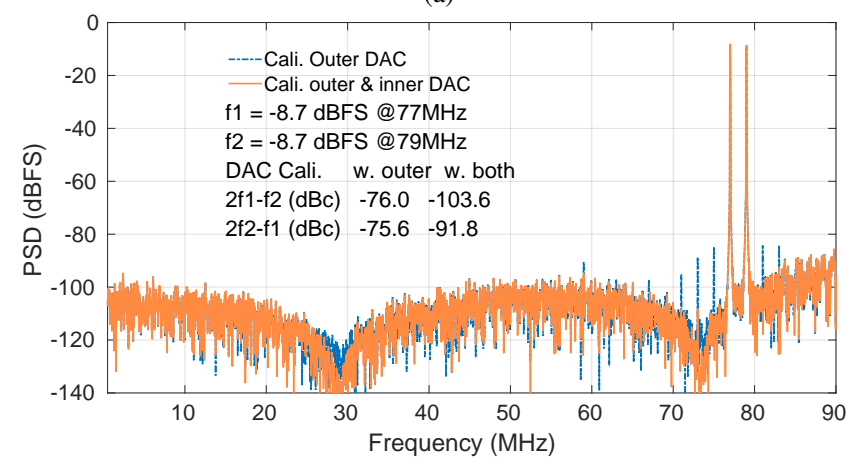

(b)

Fig. 11. Simulated two-tone spectra: (a) with only the outer $\mathrm{DAC}_{1}$ calibrated, and (b) with both the outer $\mathrm{DAC}_{1}$ and the inner $\mathrm{DAC}_{2}$ calibrated, resulting in high distortion suppression.

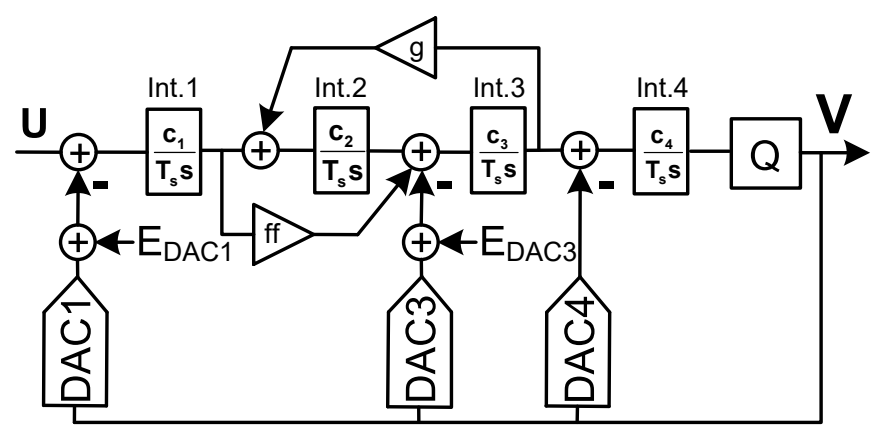

Fig. 12. The topology of the experimentally tested CIFF-B CT $\Delta \Sigma$ ADC.

shown in Fig. 12. It is the cascade-of integrators with feedforward and feedback (CIFF-B) topology. It differs from the previously described illustrative topology of Fig. 3 in the sense that $\mathrm{DAC}_{2}$ is missing. This topology was adopted because of its good tradeoff between the power consumption and the outof-band blocker immunity [8]. Designed in 28nm CMOS, it has a sampling frequency of $1.7 \mathrm{GHz}$ and an OSR of 10 , corresponding to a bandwidth of $85 \mathrm{MHz}$. A discrete-time second-order noise-shaping quantizer is used to reduce the quantization noise, without suffering from the mismatch of the signal transfer function of the multi-stage topology [13]. The quantizer has a STF of 1 and outputs 4 digital bits per sampling. The integrator time constants are trimmed to account for the $\pm 20 \%$ process variations.

All DACs have a relatively small unit size to reduce the ELD as well as the dynamic errors. In this CIFF-B topology, Int. $_{4}$ has a larger band-edge gain than Int. 3 and, similarly, the 


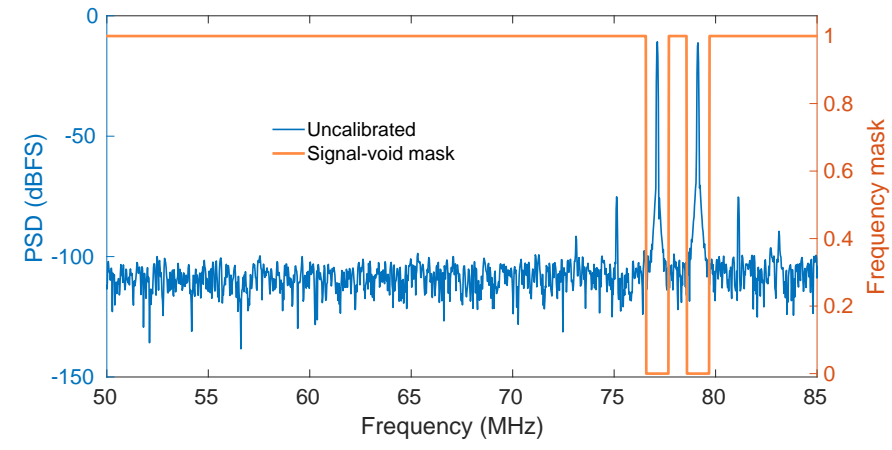

Fig. 13. Measured uncalibrated output spectrum for the $\Delta \Sigma$ ADC of Fig. 12 for a band-edge two-tone input and the applied frequency-domain mask used in the calibration.

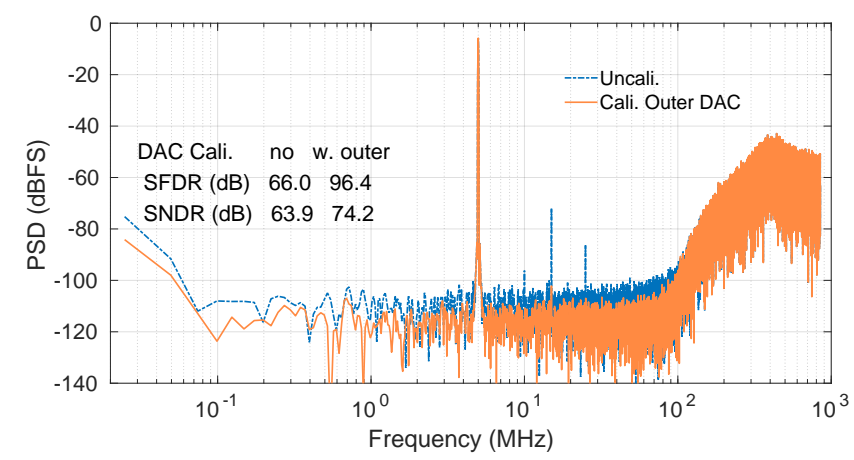

(a)

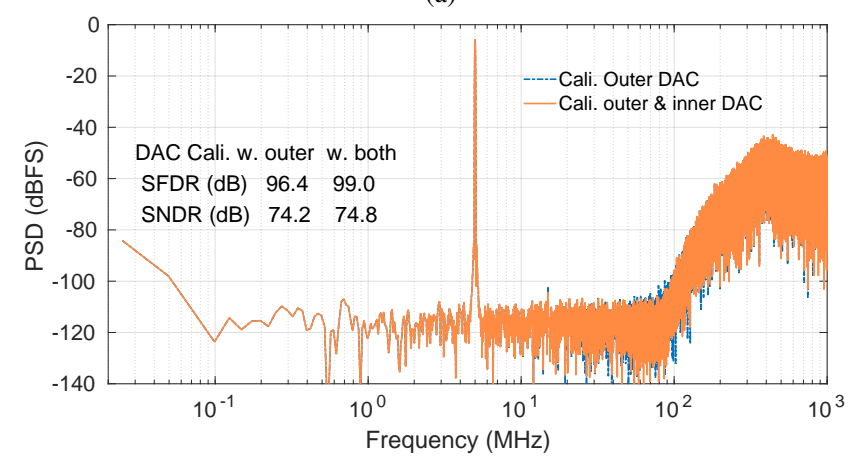

(b)

Fig. 14. Measured single-tone output spectra for the architecture of Fig. 12 for low-frequency input signals: (a) with only the outer $\mathrm{DAC}_{1}$ calibrated, and (b) with both the outer $\mathrm{DAC}_{1}$ and the inner $\mathrm{DAC}_{3}$ calibrated, showing highly reduced distortion.

band-edge gain of Int.3 is larger than that of both Int. 1 and Int.2. Hence, with the integrator coefficients for this design, the mismatch of $\mathrm{DAC}_{3}$ is relevant, while the mismatch of $\mathrm{DAC}_{4}$ is negligible. Therefore, the calibration of the experimental chip of Fig. 12 focuses on the mismatch calibration of the outer $\mathrm{DAC}_{1}$ and of the inner $\mathrm{DAC}_{3}$.

Two -10.8 dBFS input tones at 77.125 $\mathrm{MHz}$ and 79.125 $\mathrm{MHz}$ are used as excitation to calculate the errors of both DACs. The measured spectrum is shown in Fig. 13. Without any calibration, the mismatches of the DACs result in obvious intermodulation components near the actual signals. The two frequencies are chosen to make sure that all intermodulation components over $-100 \mathrm{~dB}$ fall within the bandwidth. The frequency-domain mask to void the input tones is also shown in Fig. 13. The window width where the mask is ' 0 ' around the input was chosen as $1.2 \mathrm{MHz}$ in our experiments. It should be wide enough to void the input tone and its phase noise. If the window of the mask is chosen too large, then more useful frequency information is discarded and more samples are needed to achieve the same linearity. In our test, $68 \mathrm{~K}$ samples are used to calculate the mismatches of $\mathrm{DAC}_{1}$ and $\mathrm{DAC}_{3}$, using (12).

In our experiments, we used the nominally designed filter for the first Error Transfer Function $\mathrm{ETF}_{1}$. However, for the other Error Transfer Function, $\mathrm{ETF}_{3}$, this did not give good results. After investigation we found that this was due to a mismatched delay between the actual $\mathrm{ETF}_{3}$ and the designed one, and we found that this delay had to be determined quite accurately to obtain good results. The amplitude characteristic of $\mathrm{ETF}_{3}$, on the other hand, was found to be quite uncritical and very well approximated by a simple double differentiation. In this way, we used for $\mathrm{ETF}_{3}$ the following doubledifferentiation filter, combined with a delaying phase shifter (with sub-fractional delay equal to $T_{s}\left(n_{d, i}+n_{d, f}\right)$ ) according to:

$$
\widehat{E T F}_{3}=\left(1-z^{-1}\right)^{2} \cdot z^{-n_{d, i}} \cdot \frac{1-\alpha+\alpha z^{-1}}{1+\alpha-\alpha z^{-1}}
$$

where $\alpha$ is equal to $n_{d, f} / 2$. The correct value of the total delay was found by a trial and error procedure and turned out to be equal to $4.8 T_{s}$. Note that this error transfer function enables a very simple implementation.

Fig. 14(a) plots the measured FFT for a $5 \mathrm{MHz}-4.0 \mathrm{dBFS}$ input signal with and without outer $\mathrm{DAC}_{1}$ calibration. Without calibration of the outer $\mathrm{DAC}_{1}$, the SNDR and the SFDR are limited to $63.9 \mathrm{~dB}$ and $66.0 \mathrm{~dB}$, respectively. The mismatch values of the outer $\mathrm{DAC}_{1}$ and the inner $\mathrm{DAC}_{3}$ are calculated from the measurement of Fig. 13. When only the outer $\mathrm{DAC}_{1}$ is calibrated using (14), the spectrum is shown in Fig. 14(a): the SNDR and the SFDR are significantly improved to 74.2 $\mathrm{dB}$ and $96.4 \mathrm{~dB}$, respectively. When both the outer $\mathrm{DAC}_{1}$ and the inner $\mathrm{DAC}_{3}$ are calibrated, the spectrum is shown in Fig. 14(b): the SNDR and the SFDR are even further improved to $74.8 \mathrm{~dB}$ and $99.0 \mathrm{~dB}$, respectively. At low frequencies, however, the performance improvements due to the $\mathrm{DAC}_{3}$ calibration are limited, which is due to the high attenuation of the double differentiation property of $\mathrm{ETF}_{3}$. The small residual third harmonic distortion of the calibrated spectrum is most probably due to the signal generator used.

The same calculated mismatches values have then been applied to calibrate the digital output of a high-frequency two-tone input near the band edge, as shown in Fig. 15. The uncalibrated spectrum for two $-11.5 \mathrm{dBFS}$ input tones at 70 $\mathrm{MHz}\left(\mathrm{f}_{1}\right)$ and $72 \mathrm{MHz}\left(\mathrm{f}_{2}\right)$ is shown in Fig. 15(a). Without any DAC calibration, IM2 is $-85.8 \mathrm{dBc}$ at $1 \mathrm{MHz}\left(\mathrm{f}_{2}-\mathrm{f}_{1}\right)$, and IM3 is $-64.3 \mathrm{dBc}$ at $68 \mathrm{MHz}\left(2 \mathrm{f}_{1}-\mathrm{f}_{2}\right)$ and $63.6 \mathrm{dBc}$ at 74 $\mathrm{MHz}\left(2 \mathrm{f}_{2}-\mathrm{f}_{1}\right)$. When only the outer $\mathrm{DAC}_{1}$ is calibrated, IM2 is improved to below $-100 \mathrm{dBc}$, and IM3 is improved to -79.0 $\mathrm{dBc}$ at $68 \mathrm{MHz}$ and to $-77.7 \mathrm{dBc}$ at $74 \mathrm{MHz}$. Due to the high gain of $\mathrm{ETF}_{3}$ at these near-band-edge frequencies, this first calibrated result still displays strong intermodulations, as shown in Fig. 15(a). When both the outer $\mathrm{DAC}_{1}$ and the inner 


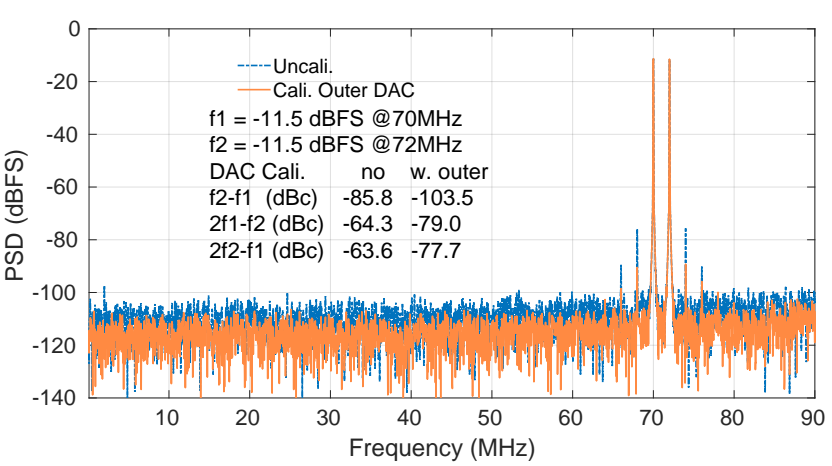

(a)

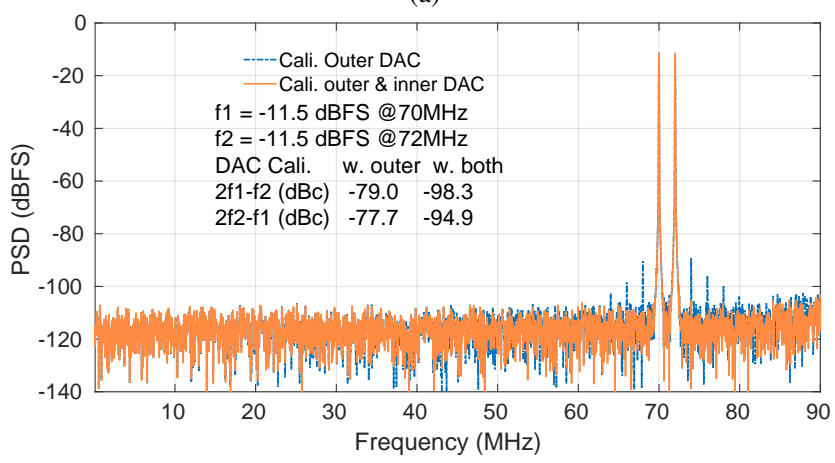

(b)

Fig. 15. Measured two-tone output spectra for the architecture of Fig. 12 for high-frequency input signals: (a) with only the outer $\mathrm{DAC}_{1}$ calibrated, and (b) with both the outer $\mathrm{DAC}_{1}$ and the inner $\mathrm{DAC}_{3}$ calibrated, showing highly reduced distortion.

$\mathrm{DAC}_{3}$ are calibrated, IM3 is further improved to $-98.3 \mathrm{dBc}$ at $68 \mathrm{MHz}$ and to $-94.9 \mathrm{dBc}$ at $74 \mathrm{MHz}$, as shown in Fig. 15(b). This clearly shows that the presented calibration method for both the outer DAC and the inner DAC drastically reduces the impact of the DAC mismatches on the ADC's nonlinearity.

\section{CONCLUSIONS}

This paper has presented an efficient frequency-domain mask-based offline mismatch calibration method for both the outer DAC and the inner DACs in continuous-time $\Delta \Sigma$ ADCs for wideband wireless communications. To avoid the need for high-performance signal generation, a frequency mask is applied to void the input signal and its phase noise. Moreover, a two-tone band-edge signal is chosen as the input stimulus during calibration. In this way, the method is robust against the harmonic distortion and phase noise in the input signal, and could be generated from two low-quality signal generators. Using the two-tone band-edge signal has the additional benefit that the number of needed samples of the excitation signal is very modest because nearly all intermodulations contribute to the calculation of the mismatches of the DACs. Simulation results have been presented that are in full agreement with the theory. They have also been confirmed by measured experimental results on a prototype chip, designed for an $85 \mathrm{MHz}$ signal bandwidth in 28nm CMOS technology. In this experiment, the calibration to calculate the DAC mismatch has been done with only $68 \mathrm{~K}$ samples. With the DACs calibrated, an SFDR improvement of $28.1 \mathrm{~dB}$ has been achieved for a single-tone input at $5 \mathrm{MHz}$, while the IM3 at $74 \mathrm{MHz}$ has been improved from $-63.6 \mathrm{dBc}$ to $-94.1 \mathrm{dBc}$ for a two-tone input around $71 \mathrm{MHz}$. The experimental results have proven the effectiveness of the proposed calibration method.

\section{ACKNOWLEDGMENT}

The authors would like to thank FWO Flanders for its financial support.

\section{REFERENCES}

[1] Erricsson, "Ericsson mobility report june 2019," [Online]. Available: https://www.ericsson.com/4a517b/assets/local/mobilityreport/documents/2019/emr-q2-2019-update.pdf.

[2] L. Breems, M. Bolatkale, H. Brekelmans, S. Bajoria, J. Niehof, R. Rutten, B. Oude-Essink, F. Fritschij, J. Singh, and G. Lassche, "A $2.2 \mathrm{GHz}$ continuous-time $\Delta \Sigma$ ADC with $-102 \mathrm{dBc}$ THD and $25 \mathrm{MHz}$ bandwidth," IEEE Journal of Solid-State Circuits, vol. 51, no. 12, pp. 2906-2916, Dec 2016.

[3] R. S. Rajan and S. Pavan, "Design techniques for continuous-time $\Delta \Sigma$ modulators with embedded active filtering," IEEE Journal of Solid-State Circuits, vol. 49, no. 10, pp. 2187-2198, Oct 2014.

[4] Y. Dong, J. Zhao, W. W. Yang, T. Caldwell, H. Shibata, Z. Li, R. Schreier, Q. Meng, J. B. Silva, D. Paterson, and J. C. Gealow, "A 72 dB-DR 465 MHz-BW continuous-time 1-2 MASH ADC in $28 \mathrm{~nm}$ CMOS," IEEE Journal of Solid-State Circuits, vol. 51, no. 12, pp. 2917 2927, Dec 2016.

[5] S. Loeda, J. Harrison, F. Pourchet, and A. Adams, "A 10/20/30/40 MHz Feedforward FIR DAC continuous-time $\triangle \Sigma$ ADC with robust blocker performance for radio receivers," IEEE Journal of Solid-State Circuits, vol. 51, no. 4, pp. 860-870, April 2016.

[6] T. Caldwell, D. Alldred, and Z. Li, "A reconfigurable $\Delta \Sigma$ ADC with up to $100 \mathrm{MHz}$ bandwidth using flash reference shuffling," IEEE Transactions on Circuits and Systems I: Regular Papers, vol. 61, no. 8, pp. 2263-2271, Aug 2014.

[7] F. Conzatti, L. Dorrer, P. Torta, C. Kropf, D. Patzold, J. S. P. Garcia, V. Rallos, and N. Schembera, "A CT $\Delta \Sigma$ ADC with $9 / 50 \mathrm{MHz}$ BW achieving $73 / 71 \mathrm{~dB}$ DR designed for robust blocker tolerance in $14 \mathrm{~nm}$ FinFET," in ESSCIRC 2017 - 43rd IEEE European Solid State Circuits Conference, Sep. 2017, pp. 139-142.

[8] S. Pavan, R. Schreier, and G. C. Temes, Understanding Delta-Sigma Data Converters. New Your, NY, USA: John Wiley \& Sons, 2017.

[9] R. Ritter and M. Ortmanns, "Continuous-time Delta-Sigma ADCs with improved interferer rejection," IEEE Journal on Emerging and Selected Topics in Circuits and Systems, vol. 5, no. 4, pp. 500-513, Dec 2015.

[10] Y. Dong, R. Schreier, W. Yang, S. Korrapati, and A. Sheikholeslami, "A $235 \mathrm{~mW}$ CT 0-3 MASH ADC achieving -167dBFS/Hz NSD with $53 \mathrm{MHz}$ BW," in 2014 IEEE International Solid-State Circuits Conference Digest of Technical Papers (ISSCC), Feb 2014, pp. 480-481.

[11] R. T. Baird and T. S. Fiez, "Linearity enhancement of multibit $\Delta \Sigma \mathrm{A} / \mathrm{D}$ and D/A converters using data weighted averaging," IEEE Transactions on Circuits and Systems II: Analog and Digital Signal Processing, vol. 42, no. 12, pp. 753-762, Dec 1995.

[12] D. W. J. Groeneveld, H. J. Schouwenaars, H. A. H. Termeer, and C. A. A. Bastiaansen, "A self-calibration technique for monolithic high-resolution D/A converters," IEEE Journal of Solid-State Circuits, vol. 24, no. 6, pp. 1517-1522, Dec 1989.

[13] D. Yoon, S. Ho, and H. Lee, "A continuous-time sturdy-MASH $\Delta \Sigma$ modulator in $28 \mathrm{~nm}$ CMOS," IEEE Journal of Solid-State Circuits, vol. 50, no. 12, pp. 2880-2890, Dec 2015.

[14] T. He, M. Ashburn, S. Ho, Y. Zhang, and G. Temes, "A 50MHZ-BW continuous-time $\Delta \Sigma$ ADC with dynamic error correction achieving 79.8 dB SNDR and 95.2 dB SFDR," in Proc. IEEE Int. Solid State Circuits Conf. (ISSCC). IEEE, 2018, pp. 230-232.

[15] M. Pelgrom, Analog-to-Digital Conversion, third edition. New York, NY, USA: Springer, 2017, pp. 273-283.

[16] S. Ho, C. Lo, J. Ru, and J. Zhao, "A $23 \mathrm{~mW}, 73 \mathrm{~dB}$ dynamic range, $80 \mathrm{MHz}$ BW continuous-time delta-sigma modulator in $20 \mathrm{~nm}$ CMOS," IEEE Journal of Solid-State Circuits, vol. 50, no. 4, pp. 908-919, April 2015.

[17] T. Cataltepe, A. R. Kramer, L. E. Larson, G. C. Temes, and R. H. Walden, "Digitally corrected multi-bit sigma delta data converters," in IEEE International Symposium on Circuits and Systems, , 1989, pp. 647650 vol.1. 
[18] J. Zhao, Y. Dong, W. Yang, H. Shibata, P. Shrestha, Z. Li, T. Caldwell, J. B. D. Silva, and J. Gealow, "A -89-dBc IMD3 DAC sub-system in a $465-\mathrm{MHz}$ BW CT delta-sigma ADC using a power and area efficient calibration technique," IEEE Transactions on Circuits and Systems II: Express Briefs, vol. 65, no. 7, pp. 859-863, July 2018.

[19] H. Liu, X. Xing, and G. G. E. Gielen, "An 85-MHz-BW ASAR-assisted CT 4-0 MASH $\Delta \Sigma$ modulator with background half-range ditheringbased DAC calibration in 28-nm CMOS," IEEE Transactions on Circuits and Systems I: Regular Papers, vol. 66, no. 7, pp. 2405-2414, July 2019.

[20] M. De Bock, X. Xing, L. Weyten, G. Gielen, and P. Rombouts, "Calibration of DAC mismatch errors in $\Sigma \Delta$ ADCs based on a sinewave measurement," IEEE Transactions on Circuits and Systems II: Express Briefs, vol. 60, no. 9, pp. 567-571, Sep. 2013.

[21] M. De Bock, A. Babaie-Fishani, and P. Rombouts, "Improved offline calibration for DAC mismatch in low OSR Sigma Delta ADCs with distributed feedback," in Proc. IEEE Int. Symp. Circuits and Syst. (ISCAS), 2014, pp. 938-941.

[22] S. Pavan and R. Theertham, "Improved offline calibration of DAC mismatch errors in delta-sigma data converters," IEEE Transactions on Circuits and Systems II: Express Briefs, vol. 66, no. 10, pp. 1618-1622, Oct 2019.

[23] J. G. Kauffman, P. Witte, M. Lehmann, J. Becker, Y. Manoli, and M. Ortmanns, "A $72 \mathrm{~dB}$ DR, CT delta sigma modulator using digitally estimated, auxiliary DAC linearization achieving $88 \mathrm{fJ} / \mathrm{conv}$-step in a 25 MHz BW," IEEE J. Solid-State Circuits, vol. 49, no. 2, pp. 392-404, Feb. 2014.

[24] J. G. Kauffman, P. Witte, J. Becker, and M. Ortmanns, "An $8.5 \mathrm{mw}$ continuous-time $\Delta \Sigma$ modulator with $25 \mathrm{MHz}$ bandwidth using digital background dac linearization to achieve $63.5 \mathrm{~dB}$ SNDR and $81 \mathrm{~dB}$ SFDR," IEEE Journal of Solid-State Circuits, vol. 46, no. 12, pp. 2869 2881, 2011.

[25] P. Witte and M. Ortmanns, "Background dac error estimation using a pseudo random noise based correlation technique for sigma-delta analogto-digital converters," IEEE Transactions on Circuits and Systems I: Regular Papers, vol. 57, no. 7, pp. 1500-1512, 2010.

[26] B. Wu, S. Zhu, B. Xu, and Y. Chiu, "A $24.7 \mathrm{~mW} 65 \mathrm{~nm}$ CMOS SARassisted CT $\Delta \Sigma$ modulator with second-order noise coupling achieving $45 \mathrm{MHz}$ bandwidth and $75.3 \mathrm{~dB}$ SNDR," IEEE Journal of Solid-State Circuits, vol. 51, no. 12, pp. 2893-2905, Dec 2016.

[27] W. Wang, C. Chan, Y. Zhu, and R. P. Martins, "A 72.6dB-SNDR $100 \mathrm{MHz}-\mathrm{BW} 16.36 \mathrm{~mW}$ CTDSM with preliminary sampling and quantization scheme in backend subranging QTZ," in 2019 IEEE International Solid-State Circuits Conference - (ISSCC), Feb 2019, pp. 340-342.

[28] R. Schreier, "Delta-sigma toolbox-high-level design and simulation of delta-sigma modulators," Matlab Toolbox. Available online: http://www. mathworks. com/matlabcentral/fileexchange/19-delta-sigma-toolbox (accessed on 29 December 2011), 2013.

[29] B. Porat, A course in digital signal processing. Wiley New York, 1997, vol. 1 .

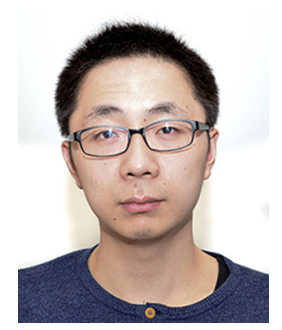

Hui Liu received the B.S. degree in automation from Harbin Institute of Technology, Harbin, China, in 2011, the M.S. degree in integrated circuit engineering from Tsinghua University, Beijing, China, in 2014. His master's topic is about readout circuit design for image sensor.

In 2016, he joined the MICAS Research Group of KU Leuven, Leuven, Belgium, where he is currently a research assistant pursuing the Ph.D. degree. His research field is about high-speed high-bandwidth $\Delta \Sigma$ modulator

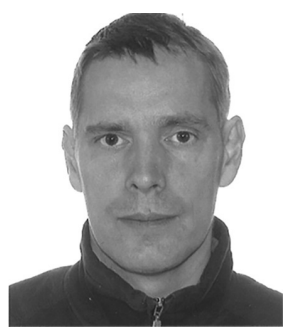

Pieter Rombouts was born in Leuven, Belgium, in 1971. He received the Ir. degree in applied physics and the Ph.D. degree in electronics from Ghent University in 1994 and 2000, respectively.Since 1994, he has been with the Electronics and Information Systems Department, Ghent University,where he has been a Professor of analog electronics since 2005.

His technical interests are signal processing, circuits and systems theory, analog circuit design, and sensor systems. The main focus of his research has been on A/D- and D/A-conversion. He has served or is currently serving as an Associate Editor for the IEEE TRANSACTIONS ONCIRCUITS ANDSYSTEMS-I, the IEEE TRANSACTIONS ONCIRCUITS ANDSYSTEMS-II, and Electronics Letters.

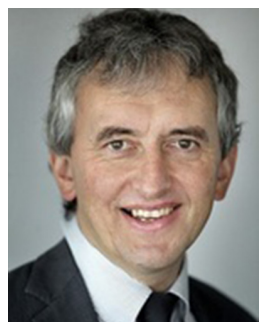

Georges G.E. Gielen (M'91-SM'00-F'02) received the M.Sc. and Ph.D. degrees in electrical engineering from KU Leuven, Leuven, Belgium, in 1986 and 1990, respectively.

From 2013 until 2017, he was also appointed as a Vice-Rector for the Group Science, Engineering and Technology, where he was responsible for academic HRM at KU Leuven. In 2018, he was elected as member of the Academia Europaea. He is currently a Full Professor with the Department of Electrical Engineering (ESAT). He has authored or co-authore seven books and over 600 papers in edited books, international journals, and conference proceedings. His research interests include the design of analog and mixed-signal integrated circuits, and especially in analog and mixed-signal $\mathrm{CAD}$ tools and design automation. He is a frequent invited speaker/lecturer and coordinator/partner of several (industrial) research projects in this area, including several European projects. 